\title{
An observation on the module structure of block algebras
}

\section{Matthew J. K. Gelvin}

To cite this article: Matthew J. K. Gelvin (2019) An observation on the module structure of block algebras, Communications in Algebra, 47:12, 5286-5293, DOI: 10.1080/00927872.2019.1617874

To link to this article: https://doi.org/10.1080/00927872.2019.1617874

曲 Published online: 26 May 2019.

Submit your article to this journal 준

Џ Article views: 61

Q View related articles $₫$

View Crossmark data 


\title{
An observation on the module structure of block algebras
}

\author{
Matthew J. K. Gelvin \\ Department of Mathematics, Bilkent University, Ankara, Turkey
}

\begin{abstract}
Let $B$ be a $p$-block of the finite group $G$. We observe that the $p$-fusion of $G$ constrains the module structure of $B$ : Any basis of $B$ that is closed under the left and right multiplications of a chosen Sylow $p$-subgroup $S$ of $G$ must in fact form a semicharacteristic biset for the fusion system on $S$ induced by $G$. The parameterization of such semicharacteristic bisets can then be applied to relate the module structure and defect theory of $B$.
\end{abstract}

\section{ARTICLE HISTORY}

Received 28 August 2018

Communicated by Sudarshan Sehgal

\section{KEYWORDS}

Blocks of finite groups; characteristic bisets;

fusion systems

\section{MATHEMATICS SUBJECT \\ CLASSIFICATION \\ 20C20; 20D20}

\section{Introduction}

Let $G$ be a finite group and $S$ a Sylow $p$-subgroup of $G$. The left and right multiplicative actions of $S$ on $G$ give a partition of $G$ by double cosets: $G=\coprod_{i=1}^{m} S g_{i} S$ for some chosen set of representatives $\left\{g_{i}\right\}$. Each double coset is a transitive $(S, S)$-biset; this partition is just the orbit decomposition of the $(S, S)$-biset ${ }_{S} G_{S}$.

If $k$ is an algebraically closed field of characteristic $p$, the group algebra $k G$ decomposes into block algebras: $k G=B_{0} \oplus B_{1} \oplus \cdots \oplus B_{n}$. Basic results in the theory of $p$-permutation modules, summarized in Proposition 2, imply that each $B_{j}$ possesses a $k$-basis $X_{j}$ that is closed under left and right $S$-multiplication. Such an $S$-invariant $k$-basis is itself an $(S, S)$-biset, and the disjoint union $\coprod_{j=0}^{n} X_{j}$ yields an $(S, S)$-biset abstractly isomorphic to ${ }_{S} G_{S}$. Thus, the $\left\{X_{j}\right\}$ can be viewed as an $(S, S)$-partition of ${ }_{S} G_{S}$ and we can group the $\left\{S g_{i} S\right\}$ so as to recover the $(S, S)$-orbit decomposition of each $X_{j}$, with the proviso that none of this is canonical.

Let us from now on focus on a particular block algebra $B$ with $S$-invariant $k$-basis $X$. The purpose of this note is to show that the $(S, S)$-biset structure of $X$ is not arbitrary, in that it is to some extent controlled by the $p$-fusion of $G$. More precisely:

Theorem 1. If $X$ is an $S$-invariant $k$-basis of $B$ and $\mathcal{F}=\mathcal{F}_{S}(G)$ is the fusion system on $S$ induced by $G$, then $X$ is an $\mathcal{F}$-semicharacteristic $(S, S)$-biset.

In Section 2, we review some basic results in the theory of $p$-permutation modules to prove the existence and uniqueness, as an $(S, S)$-biset, of an $S$-invariant $k$-basis for $B$.

In Section 3, we define semicharacteristic bisets and related notions.

In Section 4, we prove Theorem 1. In doing so, we make use of the relationship between $G$ and the algebra structure of $B$. If $b \in Z(k G)$ is the block idempotent corresponding to $B$, every $g$ 
$\in G$ commutes with $b$ and so $(g \cdot b)\left(g^{-1} \cdot b\right)=b$. As $b$ is the identity element of $B$, the assignment $g \mapsto g \cdot b$ yields a group map $G \rightarrow B^{\times}$. This makes $B$ an interior G-algebra.

We use this fact repeatedly and without further comment beyond a point on notation: Multiplication in our algebras is indicated by concatenation of elements, while the symbol "." is reserved for the action of an element of a group on an element of an algebra. (We will occasionally use " $\odot$ " for the same, when multiple group actions must be compared.) For $g \in G$, we write $\bar{g}$ for the image of $g$ in the unit group of the interior $G$-algebra $A$, so that by definition $g \cdot a=\bar{g} a$ for all $a \in A$.

Finally, in Section 5 we combine Theorem 1 with the parameterization of $\mathcal{F}$-semicharacteristic bisets from [6] to impose constraints on the $(k S, k S)$-bimodule structure of $B$. We also use the main observation to give a new perspective on a few basic results in the theory of defect groups of blocks.

Thanks are due to Laurence Barker and Justin Lynd, whose independent collaborations with the author suggested the main result of this note as an observation of potential interest.

\section{2. $p$-Permutation modules}

Let $H$ be a finite group and $M$ a finite dimensional $k H$-module. $M$ is a p-permutation $H$-module if for any $p$-subgroup $P \leq H, M$ possesses a $k$-basis $Y=Y_{P}$ that is closed under the action of $P$. Such a basis will be called $P$-invariant. $Y$ is a finite $P$-set and as such can be written

$$
Y \cong \coprod_{[Q] \leq P} c_{Q}(Y) \cdot[P / Q]
$$

where the coproduct is indexed by the $P$-conjugacy classes of subgroups of $P, c_{Q}(Y) \in \mathbb{N}$, and $[P / Q]$ denotes the transitive $P$-set having a point with stabilizer $Q$. As $k P$-modules,

$$
M \cong \bigoplus_{[Q] \leq P} c_{Q}(Y) \cdot k[P / Q]
$$

Green showed [7, Lemma 2.3a] that each $k[P / Q]$ is indecomposable as a $k P$-module, and that moreover if $k[P / Q] \cong k[P / R]$ as $k P$-modules then $[P / Q] \cong[P / R]$ as $P$-sets. The Krull-Schmidt theorem then implies:

Proposition 2. Let $H$ be a finite group, $M$ a p-permutation $H$-module, and $P \leq H$ a p-subgroup.

(i) If $Y$ and $Y^{\prime}$ are P-invariant $k$-bases of $M$, then $Y \cong Y^{\prime}$ as $P$-sets.

(ii) If $N$ is a direct summand of $M$, then $N$ is a p-permutation $H$-module.

(iii) If $N$ is a direct summand of $M$ and $Z \subset N, Y \subset M$ are P-invariant $k$-bases, then $Z$ is isomorphic to a P-subset of $Y$.

We apply these results to the $(G \times G)$-module $k G$ with action given by $\left(g_{1}, g_{2}\right) \cdot x:=\overline{g_{1}} x \overline{g_{2}}-1$. The natural $k$-basis $G$ of $k G$ is clearly closed under this action, and hence is also closed under the action restricted to any $p$-subgroup of $G \times G$. Thus $k G$ is a $p$-permutation $(G \times G)$-module.

If $b$ is a block idempotent of $k G$, we have $k G \cong k G b \oplus k G(1-b)$ as $k G$-modules. In particular, the corresponding block algebra $B=k G b$ is a direct summand of $k G$, so $B$ is a p-permutation $G$ module by Proposition 2(ii). In particular, for $S \in \operatorname{Syl}_{p}(G)$, there is an $(S \times S)$-invariant $k$-basis $X$ of $B$. The $(S \times S)$-action on $X$ is equivalent to endowing $X$ with the structure of an $(S, S)$-biset. We will freely move between these notions without comment. When viewed as an $(S, S)$-biset, $X$ is our $S$-invariant $k$-basis of $B$.

We summarize the implications of Proposition 2: 
Proposition 3. If $B$ is a block algebra of $k G$ and $S \in \operatorname{Syl}_{p}(G)$, then $B$ possesses an $S$-invariant $k$ basis X. Such an $S$-invariant $k$-basis is uniquely determined up to isomorphism of $(S, S)$-bisets. Moreover, $X$ is isomorphic to an $(S, S)$-subbiset of ${ }_{S} G_{S}$.

\section{3. $\mathcal{F}$-Semicharacteristic bisets}

Let $\mathcal{F}=\mathcal{F}_{S}(G)$ be the fusion system on $S$ induced by $G: \mathcal{F}$ is the category whose objects are the subgroups of $S$ and whose homsets are given by

$$
\mathcal{F}(P, Q)=\operatorname{Hom}_{G}(P, Q)=\left\{\varphi: P \rightarrow Q \mid \exists g \in G \text { such that } \varphi=\left.c_{g}\right|_{P}\right\},
$$

where $c_{g}: G \rightarrow G: x \mapsto g x g^{-1}$ is (left) conjugation by $g$.

An $\mathcal{F}$-characteristic biset is an abstraction of the natural $(S, S)$-biset ${ }_{S} G_{S}$ that controls the structure of $\mathcal{F}$. Some terminology is needed for the definition; let $\Omega$ be a finite $(S, S)$-biset in what follows.

The opposite biset of $\Omega$ is the $(S, S)$-biset $\Omega^{\circ}$ whose underlying set is $\Omega$ and whose left and right $S$ actions are given by $s_{1} \odot \omega \odot s_{2}:=s_{2}^{-1} \cdot \omega \cdot s_{1}^{-1}$. We say $\Omega$ is symmetric if $\Omega \cong \Omega^{\circ}$ as $(S, S)$-bisets.

The point-stabilizer of $\omega \in \Omega$ is the $(S \times S)$-stabilizer of $\omega$. This is the subgroup of $S \times S$ defined by $\operatorname{Stab}(\omega):=\left\{\left(s_{1}, s_{2}\right) \in S \times S \mid s_{1} \cdot \omega=\omega \cdot s_{2}\right\}$.

If $P \leq S$ and $\varphi: P \hookrightarrow S$ is a group monomorphism, the twisted diagonal subgroup defined by $P$ and $\varphi$ is $(\varphi, P):=\{(\varphi(u), u) \mid u \in P\} \leq S \times S$.

$\Omega$ is bifree if the left and right $S$-actions on $\Omega$ are individually free. If $\Omega$ is bifree, the point-stabilizer of every $\omega \in \Omega$ is a twisted diagonal subgroup, and we shall write $\left(c_{\omega}, S_{\omega}\right)$ for $\operatorname{Stab}(\omega)$ in this case. This notation comes from the example $\Omega={ }_{S} G_{S}$, where an element $g \in{ }_{S} G_{S}$ has point-stabilizer $\left(c_{g}, S_{g}\right)$, for $S_{g}:=S \cap S^{g}$ the largest subgroup of $S$ conjugated into $S$ by $g$.

If $P \leq S$, let ${ }_{P} \Omega_{S}$ be the $(P, S)$ biset whose left $P$-action comes from restriction of the left $S$-action. If $\varphi: P \hookrightarrow S$ is a group monomorphism, ${ }_{P}^{\varphi} \Omega_{S}$ is the $(P, S)$-biset whose left $P$-action is realized by first twisting by $\varphi$ : For all $u \in P, s \in S$, and $\omega \in \Omega$, set $u \odot \omega \odot s:=\varphi(u) \cdot \omega \cdot s$. The $(S, P)$-bisets ${ }_{S} \Omega_{P}$ and ${ }_{S} \Omega_{P}^{\varphi}$ are defined similarly.

We can now give the precise definition of $\mathcal{F}$-(semi)characteristic bisets. This notion is due to Linckelmann and Webb, who formulated it in terms of abstract saturated fusion systems. As we deal only with fusion system realized by finite groups, we shall make no further commentary on the more general situation. See, e.g., [1] for the complete picture.

Definition 4. Let $\mathcal{F}$ be a saturated fusion system on the p-group $S$ and let $\Omega$ be an $(S, S)$-biset.

- $\Omega$ is $\mathcal{F}$-generated if for all $\omega \in \Omega$ with point-stabilizer $\left(c_{\omega}, S_{\omega}\right)$, the group map $c_{\omega}: S_{\omega} \rightarrow S$ lies in $\mathcal{F}\left(S_{\omega}, S\right)$.

- $\Omega$ is $\mathcal{F}$-stable if for all $P \leq S$ and $\varphi \in \mathcal{F}(P, S)$, we have ${ }_{P}^{\varphi} \Omega_{S} \cong{ }_{P} \Omega_{S}$ as $(P, S)$-bisets and ${ }_{S} \Omega_{P}^{\varphi} \cong{ }_{S} \Omega_{P}$ as $(S, P)$-bisets.

- $\Omega$ is $\mathcal{F}$-semicharacteristic if

(i) $\Omega$ is bifree,

(ii) $\Omega$ is symmetric,

(iii) $\Omega$ is $\mathcal{F}$-generated, and

(iv) $\Omega$ is $\mathcal{F}$-stable.

- $\Omega$ is $\mathcal{F}$-characteristic if $\Omega$ is $\mathcal{F}$-semicharacteristic and in addition

(v) $|\Omega| /|S|$ is prime to $p$.

The existence of characteristic bisets for abstract saturated fusion systems was shown in [3, Proposition 5.5], though in our case ${ }_{S} G_{S}$ itself gives an example. It was also proved in [9, Proposition 21.9] that if an $\mathcal{F}$-characteristic biset exists then $\mathcal{F}$ must be saturated, albeit with different terminology. 
As noted above, ${ }_{S} G_{S}$ is, in fact, an $\mathcal{F}=\mathcal{F}_{S}(G)$-characteristic biset:

(i) ${ }_{S} G_{S}$ is bifree as an $(S, S)$-biset as both left and right multiplication in a group are invertible operations.

(ii) ${ }_{S} G_{S}$ is symmetric via the inversion map $g \mapsto g^{-1}$.

(iii) Any $g \in G$ has point-stabilizer $\left(c_{g}, S_{g}\right)$, and $c_{g} \in \mathcal{F}\left(S_{g}, S\right)$ by definition.

(iv) If $\varphi=c_{g} \in \mathcal{F}(P, S)$, left multiplication by $g$ yields ${ }_{P} G_{S} \cong{ }_{P}^{\varphi} G_{S}$ and right multiplication by $g^{-1}$ yields ${ }_{S} G_{P} \cong{ }_{S} G_{P}^{\varphi}$, so ${ }_{S} G_{S}$ is $\mathcal{F}$-stable.

(v) $|G| /|S|$ is prime to $p$ as $S$ is a Sylow $p$-subgroup of $G$.

The proof that an $S$-invariant $k$-basis $X$ of $B$ is $\mathcal{F}$-semicharacteristic will amount to a checklist verification of Conditions (i)-(iv). In Section 5, we will see that an $S$-invariant $k$-basis of $B$ satisfies Conditon (v) if and only if $B$ has maximal defect, that is, $S$ is a defect group of $B$.

\section{The proof of Theorem 1}

Only Conditions (ii) and (iv) in the definition of $\mathcal{F}$-semicharacteristic biset are not obvious for our $S$-invariant $k$-basis $X$. We prove these separately in the following two propositions.

Proposition 5. $X$ is $\mathcal{F}$-stable.

Proof. Let $P \leq S$ and $\varphi \in \mathcal{F}(P, S)$ be given. We show ${ }_{P}^{\varphi} X_{S} \cong{ }_{P} X_{S}$ as $(P, S)$-bisets; the proof that ${ }_{S} X_{P}^{\varphi} \cong{ }_{S} X_{P}$ as $(S, P)$-bisets is the same.

Fix $g \in G$ inducing $\varphi \in \mathcal{F}(P, S)$, so $g u g^{-1}=\varphi(u)$ for all $u \in P$. Set $X^{\prime}=g^{-1} \cdot X$. As $g^{-1} \cdot X=$ $\bar{g}^{-1} X$, we see that $X^{\prime}$ is a $k$-basis of $B$ multiplied by a unit. In particular, $X^{\prime}$ is also a $k$-basis of $B$.

For any $g^{-1} \cdot x \in X^{\prime}$ and $u \in P$, we have $u \cdot\left(g^{-1} \cdot x\right)=g^{-1} \cdot(\varphi(u) \cdot x)$. As $\varphi(u) \in S$ and $X$ is $S$-invariant, we conclude that $u \cdot X^{\prime}=X^{\prime}$, and thus $X^{\prime}$ is a $(P \times S)$-invariant $k$-basis of $B$. That $X^{\prime}$ is invariant under the right $S$-action is obvious. Proposition 2(i) implies that $X \cong X^{\prime}$ as $(P, S)$-bisets, say via $f: X \rightarrow X^{\prime}$. Then for all $u \in P$ and $x \in X$, the composite bijection $F: X \rightarrow X^{\prime} \rightarrow X: x \mapsto f(x) \mapsto g \cdot f(x)$ satisfies

$$
F(u \cdot x)=g \cdot f(u \cdot x)=g u \cdot f(x)=\varphi(u) \cdot(g \cdot f(x))=\varphi(u) \cdot F(x) .
$$

Again it is obvious that $F(x \cdot s)=F(x) \cdot s$ for all $s \in S$. Thus, $F$ is an isomorphism ${ }_{P} X_{S} \cong_{P}^{\varphi} X_{S}$ of $(P, S)$-bisets, so $X$ is $\mathcal{F}$-stable.

In order to prove the symmetry of $X$, we make a small detour.

Let $A$ be a finite dimensional $k$-algebra. A symmetrizing form on $A$ is a $k$-linear map $\lambda: A \rightarrow k$ whose kernel contains no nontrivial left (or right) ideals and such that $\lambda\left(a_{1} a_{2}\right)=\lambda\left(a_{2} a_{1}\right)$ for all $a_{1}, a_{2} \in A$. If $A$ possesses a symmetrizing form, $A$ is a symmetric $k$-algebra. Equivalently, $A$ is a symmetric $k$-algebra if the regular $(A, A)$-bimodule ${ }_{A} A_{A}$ is isomorphic to its linear dual $A^{*}:=$ $\operatorname{Hom}_{k}(A, k)$ as $(A, A)$-bimodules (see, e.g., [10, Section 1.6] for a review of this material, and a more general version of Lemma 7.)

For $H$ a finite group, the $k$-algebra $A$ is an interior p-permutation $H$-algebra if $A$ is an interior $H$-algebra and for any $p$-subgroup $P \leq H, A$ possesses a $P$-invariant $k$-basis $Y=Y_{P}$.

Proposition 6. Let $A$ be a symmetric interior p-permutation $H$-algebra. If $P \leq H$ is a p-subgroup and $Y$ is a $P$-invariant $k$-basis of $A$, then $Y$ is symmetric as a $(P, P)$-biset.

Proof. Enumerate $Y=:\left\{y_{1}, y_{2}, \ldots, y_{n}\right\}$, and let $Y^{*}=\left\{y_{1}^{*}, y_{2}^{*}, \ldots, y_{n}^{*}\right\}$ be the dual basis of $A^{*}$ with respect to $Y$, that is, $y_{i}^{*}\left(y_{j}\right)=\delta_{i j}$.

Let $\lambda$ be a symmetrizing form of $A$. For each $a \in A$, let $\lambda_{a}: A \rightarrow k$ be the linear functional $\lambda_{a}: a^{\prime} \mapsto \lambda\left(a a^{\prime}\right)$. Clearly the assignment $a \mapsto \lambda_{a}$ defines a $k$-linear map $A \rightarrow A^{*}$. If $\lambda_{a}$ is the trivial functional for some $a \in A$, then $\lambda(A a)=\lambda(a A)=0$, so the left ideal $A a$ is contained in the 
kernel of $\lambda$. The assumption that ker $\lambda$ contains no nontrivial left ideals forces $a=0$, so $\lambda_{-}: A \rightarrow$ $A^{*}$ is a $k$-injection. As $A$ is finite dimensional over $k$, we conclude that $\lambda_{-}$is a $k$-isomorphism.

Thus, for each $y_{i} \in Y$, there is a unique $\check{y}_{i} \in A$ such that $\lambda_{\check{y}_{i}}=y_{i}^{*}$. In other words, $\check{y}_{i}$ is defined by $\lambda\left(\check{y}_{i} y_{j}\right)=\delta_{i j}$. Set $\check{Y}:=\left\{\check{y}_{1}, \check{y}_{2}, \ldots, \check{y}_{n}\right\}$. Clearly $\check{Y}$ is a $k$-basis for $A$.

Consider now that, for $u_{1}, u_{2} \in P, \check{y}_{i} \in \check{Y}$, and $y_{j} \in Y$, we have

$$
\lambda\left(\left(u_{1} \cdot \check{y}_{i} \cdot u_{2}\right) y_{j}\right)=\lambda\left(\bar{u}_{1} \check{y}_{i} \bar{u}_{2} y_{j}\right)=\lambda\left(\check{y}_{i} \bar{u}_{2} y_{j} \bar{u}_{1}\right)=\lambda\left(\check{y}_{i}\left(u_{2} \cdot y_{j} \cdot u_{1}\right)\right) .
$$

As $Y$ is $P$-invariant, $u_{2} \cdot y_{j} \cdot u_{1} \in Y$, so the above common value is 1 if $u_{2} \cdot y_{j} \cdot u_{1}=y_{i}$, or equivalently $y_{j}=u_{2}^{-1} \cdot y_{i} \cdot u_{1}^{-1}$, and 0 otherwise. Thus

$$
u_{1} \cdot \check{y}_{i} \cdot u_{2}=\left(u_{2}^{-1} \cdot y_{i} \cdot u_{1}^{-1}\right)^{\vee} \in \check{Y} .
$$

This shows both that $\check{Y}$ is $P$-invariant and that $\check{Y} \cong Y^{\circ}$ as $(P, P)$-bisets.

As $\check{Y}$ is a $P$-invariant $k$-basis of $A$, Proposition $2(\mathrm{i})$ implies $Y \cong \check{Y}$ as $(P, P)$-bisets as well. Combining these isomorphisms gives $Y \cong Y^{\circ}$, so $Y$ is a symmetric $(P, P)$-biset.

The last necessary ingredient is that algebra direct summands of symmetric algebras are symmetric:

Lemma 7. Let $A$ be a symmetric $k$-algebra with symmetrizing form $\lambda$. If $e \in A$ is idempotent, then eAe is symmetric with symmetrizing form $\left.\lambda\right|_{e A e}$.

Proof. Let $\bar{\lambda}=\left.\lambda\right|_{e A e}$. Clearly $\bar{\lambda}(x y)=\bar{\lambda}(y x)$ for all $x, y \in e A e$, so it suffices to show that $\bar{\lambda}$ contains no nonzero left ideals of $e A e$.

Let $J \subseteq e A e$ be a left ideal of $e A e$ contained in $\operatorname{ker} \bar{\lambda}$. As $e$ is the identity element of $e A e$, we have $J=e J e$. Consider the left ideal $A J$ of $A$ generated by $J$. Then we have

$$
\lambda(A J)=\lambda(\text { AeJe })=\lambda(e A e J)=\lambda(J)=\bar{\lambda}(J)=0,
$$

so that $A J$ is a left $A$-ideal contained in ker $\lambda$. Thus, $J \subseteq A J=0$, and we have verified that $\bar{\lambda}$ is a symmetrizing form for $e A e$.

Proof of Theorem 1. Let $X$ be an $S$-invariant $k$-basis for the block $B$, whose existence is guaranteed by Proposition 3. Proposition 2(iii) implies that $X$ is isomorphic to an $(S, S)$-subbiset of ${ }_{S} G_{S}$, which we have already observed to be $\mathcal{F}$-characteristic. Bifreeness and $\mathcal{F}$-generation are clearly properties inherited by $(S, S)$-subbisets, so we have verified Conditions (i) and (iii) in the definition of $\mathcal{F}$-semicharacteristic bisets.

The group algebra $k G$ is symmetric with symmetrizing form $\lambda: \sum_{g \in G} \alpha_{g} g \mapsto \alpha_{1}$. The block algebra $B=k G b=b(k G) b$ is the group algebra cut by an idempotent, so $B$ is symmetric by Lemma 7. Therefore $B$ is a symmetric interior $p$-permutation $S$-algebra, so $X$ is symmetric by Proposition 6, and we have satisfied Condition (ii).

Finally, $X$ is $\mathcal{F}$-stable by Proposition 5, which verifies Condition (iv). This completes the proof that $X$ is $\mathcal{F}$-semicharacteristic.

\section{Some implications}

In [6] it is shown that the monoid of $\mathcal{F}$-semicharacteristic bisets possesses a natural basis $\left\{\Omega_{P}^{\mathcal{F}}\right\}$, indexed by the $\mathcal{F}$-conjugacy classes of subgroups of $S$. In particular, the $S$-invariant $k$-basis $X$ of $B$ decomposes uniquely in terms of this basis, which significantly constrains the $(S, S)$-biset structure of $X$. We recall the characterization of this basis now:

If $(\psi, Q)$ is a twisted diagonal subgroup of $S \times S$, let $[\psi, Q]$ denote the transitive $(S, S)$-biset that contains an element with point stabilizer $(\psi, Q)$. For $\Omega$ an $\mathcal{F}$-semicharacteristic biset, Conditions 
(i) and (iii) imply

$$
\Omega \cong \coprod_{\substack{Q \leq S \\ \psi \in \mathcal{F}(Q, S)}} c_{(\psi, Q)}(\Omega) \cdot[\psi, Q]
$$

for some uniquely determined $c_{(\psi, P)}(\Omega) \in \mathbb{N}$.

Let $P \leq S$ be fully $\mathcal{F}$-normalized, which in our case $\mathcal{F}=\mathcal{F}_{S}(G)$ means $N_{S}(P) \in \operatorname{Syl}_{p}\left(N_{G}(P)\right)$. Then by [6, Theorems 4.5 and 5.3] there is a unique $\mathcal{F}$-semicharacteristic biset $\Omega_{P}^{\mathcal{F}}$ such that $c_{(\mathrm{id}, P)}\left(\Omega_{P}^{\mathcal{F}}\right)=1$ and if $P^{\prime}$ is any fully $\mathcal{F}$-normalized subgroup with $c_{\left(\mathrm{id}, P^{\prime}\right)}\left(\Omega_{P}^{\mathcal{F}}\right) \neq 0$ we have $P^{\prime} \cong_{\mathcal{F}} P$. Moreover, if $c_{(\psi, Q)}\left(\Omega_{P}^{\mathcal{F}}\right) \neq 0$ then $(\psi, Q)$ is $(\mathcal{F} \times \mathcal{F})$-subconjugate to (id, $\left.P\right)$, that is, there exist $\chi \in$ $\mathcal{F}(Q, P)$ and $\chi^{\prime} \in \mathcal{F}(\psi(Q), P)$ with $\chi=\chi^{\prime} \circ \psi$.

If $[\mathrm{Cl}(\mathcal{F})]_{f n}$ is a chosen set of fully $\mathcal{F}$-normalized representatives of the $\mathcal{F}$-conjugacy classes of subgroups of $S,\left\{\Omega_{P}^{\mathcal{F}} \mid P \in[C l(\mathcal{F})]_{f n}\right\}$ is a basis for the monoid of $\mathcal{F}$-semicharacteristic bisets. Thus, any $\mathcal{F}$-semicharacteristic biset $\Omega$ can be uniquely written

$$
\Omega \cong \coprod_{P \in[C l(\mathcal{F})]_{f n}} c_{P}(\Omega) \cdot \Omega_{P}^{\mathcal{F}}
$$

This applies in particular to the case $\Omega=X$ under consideration.

Even more information can be obtained through consideration of the Brauer map. We recall basic facts from the literature without proof; see, e.g., [10] for a full treatment.

If $A$ is an interior $G$-algebra and $H \leq G$, the $H$-fixed subalgebra of $A$ is $A^{H}:=\left\{a \in A \mid h \cdot a \cdot h^{-1}=\right.$ $a \forall h \in H\}$. If $K \leq H$, let $\operatorname{tr}_{K}^{H}: A^{K} \rightarrow A^{H}$ denote the trace map $a \mapsto \sum_{h \in[H / K]} h \cdot a \cdot h^{-1}$, where $[H / K]$ is a chosen set of coset representatives of $H / K$. The subalgebra $A_{\ddagger}^{H}:=\sum_{K \nsucceq H} \operatorname{tr}_{K}^{H}\left(A^{K}\right)$ is an ideal of $A^{H}$. The Brauer quotient of $A$ at $H$ is $A(H):=A^{H} / A_{\ddagger}^{H}$ and the Brauer map of $A$ at $H$ is the natural surjection $\mathrm{br}_{H}: A^{H} \rightarrow A(H)$. Note that $A(H)=0$ unless $H$ is a $p$-subgroup of $G$.

In the special case that $A=k G$ and $P \leq G$ is a $p$-subgroup, we have $A(P) \cong k C_{G}(P)$. This reflects the more general fact that if $A$ is an interior $p$-permutation $G$-algebra, $P \leq G$ is a $p$-subgroup, and $Y$ a $P$-invariant $k$-basis of $A$, then the image of $Y^{P}:=Y \cap A^{P}$ under the Brauer map is a $k$-basis for $A(P)$. In particular, $Y^{P} \neq \emptyset$ if and only if $A(P) \neq 0$.

If $A^{G}$ is local (e.g., our block algebra $B$ ), a defect group of $A$ is a maximal $p$-subgroup $D \leq G$ such that $A(D) \neq 0$. Defect groups are well-defined up to G-conjugacy and if $P \leq G$ is any $p$-subgroup with $A(P) \neq 0$, then $P$ is $G$-subconjugate to $D$.

Putting all this together, we obtain:

Corollary 8. Let $X$ be an S-invariant k-basis of the block algebra $B$ with defect group $D \in[C l(\mathcal{F})]_{f n}$. Then X contains a copy of $\Omega_{P}^{\mathcal{F}}$ only if $P \leq{ }_{\mathcal{F}} D$, and the number of copies of $\Omega_{D}^{\mathcal{F}}$ contained in $X$ is prime to $p$.

Proof. Suppose that $X$ contains a copy of $\Omega_{P}^{\mathcal{F}}$, which in turn contains the $(S, S)$-orbit $[\mathrm{id}, P]$. As $[\text { id, } P]^{P} \neq \emptyset$, we have $B(P) \neq 0$. The characterization of defect groups then implies the first claim.

For the second claim, note that the $(S, S)$-orbit $[\psi, Q]$ has order $|S|^{2} /|Q|$, and if $\Omega_{P}^{\mathcal{F}}$ contains the orbit $[\psi, Q]$ then $(\psi, Q)$ is $(\mathcal{F} \times \mathcal{F})$-subconjugate to $(\mathrm{id}, P)$. In particular $|Q| \leq|P|$, and we conclude that $|S|^{2} /|P|$ divides $\left|\Omega_{P}^{\mathcal{F}}\right|$.

By [4, Theorem 1], the greatest power of $p$ that $\operatorname{divides} \operatorname{dim}_{k}(B)$ is $|S|^{2} /|D|$. If $P \in[C l(\mathcal{F})]_{f n}$ is such that $X$ contains a copy of $\Omega_{P}^{\mathcal{F}}$, we have $P \leq{ }_{\mathcal{F}} D$. For all such $P$ of order strictly less than $|D|$ the size of $\Omega_{P}$ has $p$-part strictly greater than $|S|^{2} /|D|$. It follows that the number of copies of $\Omega_{D}^{\mathcal{F}}$ in $X$ is prime to $p$.

In particular, $B$ has an $\mathcal{F}$-characteristic $S$-invariant $k$-basis if and only $B$ is of maximal defect, as claimed at the end of Section 3.

Corollary 8 can also be seen to imply some basic facts in the literature: 
- In [8, Theorem 3] it is proved that a defect group $D$ of $B$ is a Sylow intersection subgroup, that is, $D=S \cap S^{g}$ for some $g \in G$. Moreover, $g$ can be chosen to lie in $C_{G}(D)$ :

An $S$-invariant $k$-basis of $B$ contains $\Omega_{D}^{\mathcal{F}}$, which contains [id, $D$ ]. Proposition 2(iii) then implies that ${ }_{S} G_{S}$ must contain an element $g$ with stabilizer (id, $\left.D\right)$. As $\operatorname{Stab}(g)=\left(c_{g}, S \cap S^{g}\right)$, the result follows.

- Alperin and Green (each crediting the other, cf. [2, Section 6] and [8, Theorem 4]) show that, in our terminology, $D$ can be chosen to be fully $\mathcal{F}$-normalized in $S$ :

$D$ is $G$-conjugate to $P \in[C l(\mathcal{F})]_{f n}$, which is by definition fully $\mathcal{F}$-normalized.

The sketched proofs we offer for these basic facts are morally the same as those found in [8], so we will not elaborate further. These points are raised mainly because we find it interesting that the proofs are essentially contained in the characterization of the $\mathcal{F}$-semicharacteristic biset basis $\left\{\Omega_{P}^{\mathcal{F}}\right\}$.

We conclude by using Theorem 1 to give a new perspective on some well-known results in block theory (see, e.g., [5, Corollary 3.11]). We say that $G$ is of characteristic $p$ (also known as $p$-constrained and $p$-reduced) if $C_{G}\left(O_{p}(G)\right) \leq O_{p}(G)$, and is of local characteristic $p$ if $N_{G}(P)$ is of characteristic $p$ for all nonidentity $p$-subgroups $P \leq G$.

Corollary 9. If $G$ is of characteristic $p, k G$ has a unique block. If $G$ is of local characteristic $p$, the defect groups of all nonprincipal blocks are trivial.

Proof. The first claim follows from [6, Theorem 6.7], which implies that if $G$ is of characteristic $p$ then ${ }_{S} G_{S} \cong \Omega_{S}^{\mathcal{F}}$ as $(S, S)$-bisets. In this case, ${ }_{S} G_{S}$ cannot be broken into smaller $\mathcal{F}$-semicharacteristic bisets, so the principal block $B_{0}$ must have an $S$-invariant $k$-basis isomorphic to ${ }_{S} G_{S}$. It follows that $B_{0}=k G$, and the claim is proved.

More generally, let $B$ be a block of $k G$ with defect group $D \in[C L(\mathcal{F})]_{f n}$ (which may be trivial). By Corollary 8 , the $S$-invariant $k$-basis $X$ of $B$ must contain a copy of $\Omega_{D}^{\mathcal{F}}$, which in turn implies that there is some $g \in G$ with point-stabilizer $(\mathrm{id}, D)$. This element must satisfy $D=S \cap S^{g}$ and $g \in C_{G}(D) \leq N_{G}(D)$.

As $D \leq S$ is fully $\mathcal{F}$-normalized, we have $N_{S}(D) \in \operatorname{Syl}_{p}\left(N_{G}(D)\right)$. Thus $O_{p}\left(N_{G}(D)\right) \leq$ $N_{S}(D) \cap N_{S}(D)^{g} \leq S \cap S^{g}=D$. As $D$ is a normal p-subgroup of $N_{G}(D)$, it follows that $O_{p}\left(N_{G}(D)\right)$ $=D$. Thus $g \in C_{G}\left(O_{p}\left(N_{G}(D)\right)\right.$.

If we assume now that $G$ is of local characteristic $p$, the above implies that we must have either $D=1$ or $g \in O_{p}\left(N_{G}(D)\right)=D$. In the second case, $g \in D=S \cap S^{g}$, so $D=S$. By Corollary 8 again, we see that every block with nontrivial defect group has an $S$-invariant $k$-basis that contains a copy of $\Omega_{S}^{\mathcal{F}}$, and hence an $(S, S)$-orbit isomorphic to [id, $S$ ]. The same argument shows that ${ }_{S} G_{S}$ has a unique such orbit, which is already accounted for in the $S$-invariant $k$-basis of the principal block $B_{0}$. Thus, we see that if $G$ is of local characteristic $p$, any block of $k G$ with nontrivial defect must be the principal block, and the result is proved.

\section{References}

[1] Aschbacher, M., Kessar, R., Oliver, B. (2011). Fusion Systems in Algebra and Topology, Volume 391 of London Mathematical Society Lecture Note Series. Cambridge: Cambridge University Press.

[2] Alperin, J. L. (1967). Sylow intersections and fusion. J. Algebra. 6(2):222-241. DOI: 10.1016/00218693(67)90005-1.

[3] Broto, C., Levi, R., Oliver, B. (2003). The homotopy theory of fusion systems. J. Amer. Math. Soc. 16(04): 779-856. electronic), DOI: 10.1090/S0894-0347-03-00434-X.

[4] Brauer, R. (1976). Notes on representations of finite groups. I. J. London Math. Soc. 13(1):162-166. DOI: $10.1112 / \mathrm{jlms} / \mathrm{s} 2-13.1 .162$. 
[5] Feit, W. (1982). The Representation Theory of Finite Groups, Volume 25 of North-Holland Mathematical Library. Amsterdam: North-Holland Publishing Co.

[6] Gelvin, M., Reeh, S. P. (2015). Minimal characteristic bisets for fusion systems. J. Algebra. 427:345-374. DOI: $10.1016 /$ j.jalgebra.2014.12.020.

[7] Green, J. A. (1962). Blocks of modular representations. Math. Z. 79(1):100-115. DOI: 10.1007/BF01193108.

[8] Green, J. A. (1968). Some remarks on defect groups. Math. Z. 107(2):133-150. DOI: 10.1007/BF01111026.

[9] Puig, L. (2009). Frobenius Categories versus Brauer Blocks, Volume 274 of Progress in Mathematics (The Grothendieck Group of the Frobenius Category of a Brauer Block). Basel: Birkhäuser Verlag.

[10] Thévenaz, J. (1995). G-algebras and Modular Representation Theory (Oxford Mathematical Monographs). New York: The Clarendon Press, Oxford University Press. DOI: 10.1086/ahr/100.5.1552. 